\title{
Effect of receiving a heart transplant: analysis of a national cohort entered on to a waiting list, stratified by heart failure severity
}

\author{
Mario C Deng, Johan M J De Meester, Jacqueline M A Smits, Joachim Heinecke, Hans H Scheld on \\ behalf of the Comparative Outcome and Clinical Profiles in Transplantation (COCPIT) Study Group
}

Editorial by Hunt
Department of
Cardiothoracic
Surgery, Muenster
University, D-48129
Muenster, Germany
Mario C Deng
associate professor of
medicine and
cardiology
Hans H Scheld
professor and director
Eurotransplant
Foundation,
PO Box 2304,
2301 CH Leiden,
Netherlands
Johan M J De
Meester
head of medical
affairs
Jacqueline M A
Smits
biostatistician
Institute for
Biomathematics,
Muenster
University, D-48129
Muenster, Germany
Joachim Heinecke
senior biostatistician
Correspondence to:
M C Deng, Heart
Failure Center,
Columbia
University College
of Physicians and
Surgeons, New
York, NY 10032,
USA
md785@
columbia.edu

BMJ 2000;321:540-5

\begin{abstract}
Objective To determine whether there is a survival benefit associated with cardiac transplantation in Germany.

Design Prospective observational cohort study. Setting All 889 adult patients listed for a first heart transplant in Germany in 1997.

Main outcome measure Mortality, stratified by heart failure severity.

Results Within 1 year after listing, patients with a predicted high risk had the highest global death rate (51\% v 32\% and 29\% for medium and low risk patients respectively; $\mathrm{P}<0.0001$ ), had the highest risk of dying on the waiting list (32\% $v 20 \%$ and $20 \%$; $\mathrm{P}=0.0003)$, and were more likely to receive a transplant $(48 \% v 45 \%$ and $41 \% ; \mathrm{P}=0.01)$. Differences between the risk groups in outcome after transplantation did not reach significance $(\mathrm{P}=0.2)$. Transplantation was not associated with a reduction in mortality risk for the total cohort, but it did provide a survival benefit for the high risk group.

Conclusion Cardiac transplantation in Germany is currently associated with a survival benefit only in patients with a predicted high risk of dying on the waiting list. Patients with a predicted low or medium risk have no reduction in mortality risk associated with transplantation; they should be managed with organ saving approaches rather than transplantation.
\end{abstract}

\section{Introduction}

The survival benefit of cardiac transplantation as compared with conventional treatment in advanced heart failure has not been tested in a prospective randomised trial. The main reason has probably been the assumption that, since the introduction of cyclosporin (ciclosporin) in 1980, the benefit of cardiac transplantation over conventional treatment is clinically evident. ${ }^{1}$ Meanwhile, opposing developments have occurred. On the one hand, scientific advances have improved medical and surgical treatment for advanced heart failure. ${ }^{2-4}$ On the other hand, listing of more critically ill patients and use of so called marginal donor hearts, driven by the increasing scarcity of donors, have been associated with a lack of improvement of outcomes after cardiac transplantation, ${ }^{56}$ even with better transplant management. As a result, the survival benefit of cardiac transplantation over other treatment options is less clear than it seemed 10-20 years ago. Consequently, the presently liberal practice of listing patients with heart failure for transplantation must be questioned until clear evidence of the benefits is available. This reasoning is in line with the current reassessment of treatment options for patients with end stage liver, lung, and renal disease. ${ }^{7-9}$
Since a randomised trial to test the survival benefit of cardiac transplantation would be hampered by ethical concerns, ${ }^{10}$ we performed a prospective observational study. Such a method requires the assessment of clinical and prognostic profiles in order to analyse the effect of the intention to treat by transplantation. In advanced heart failure only one validated prognostic tool is currently available-the heart failure survival score ${ }^{11}$ which is an index for predicting mortality in stable patients awaiting a heart transplant. Much as the Child-Pugh and the Mayo scores are used to guide clinical decisions at the time of listing for liver transplantation, ${ }^{12}$ we applied the heart failure survival score as an index of disease severity to our unselected cohort of patients with advanced heart failure in order to assess differences in outcomes. Specifically, we analysed global mortality, waiting list mortality, and mortality after transplantation as well as the effect of cardiac transplantation on survival for the first time in a complete national cohort of candidates for heart transplantation stratified by disease severity at the time of listing.

\section{Patients and methods}

Patients, parameters, and data acquisition

The study originated in a consensus decision of the Heart Committee of the German Transplantation Society and the Eurotransplant International Foundation (Eurotransplant). Eurotransplant is the organ exchange organisation in which transplant centres in Austria, Belgium, Luxembourg, Germany, and the Netherlands collaborate with their donor hospitals and tissue typing laboratories. The goal was to create a continuous database to monitor quality of care in compliance with the new German transplant law, which was enacted in November 1997. The project was named the "comparative outcomes and clinical profiles in transplantation (COCPIT)" study.

All adults aged 16 or over who were consecutively listed for cardiac transplantation between 1 January and 31 December 1997 in any one of the centres then performing cardiac transplantation in Germany were included (see complete list of centres in the appendix). Data were transmitted to Eurotransplant by every centre and entered into a central database. Allocation of heart transplants was in agreement with official Eurotransplant allocation rules at the time. In brief, the rules combine medical urgency, geography, matching of donor and recipient body length, matching of $\mathrm{ABO}$ blood group, and cumulative waiting time. Besides the category of general urgency, centres were allowed special urgency requests to Eurotransplant for up to $15 \%$ of their transplantations for patients considered very sick. They were also allowed high urgency requests 
to Eurotransplant for patients with acute graft failure and, consequently, in urgent need of retransplantation.

\section{Heart failure survival score}

The original heart failure survival score was derived and validated between 1986 and 1995 in two US cohorts of stable outpatient candidates for a cardiac transplant. ${ }^{11}$ It is the weighted sum of seven non-invasive clinical parameters, the weighting reflecting the strength and direction of the association between each parameter and outcome. Outcome was defined as composite end points of death on the waiting list and urgent transplantation. These parameters were the presence of coronary artery disease (impact of aetiology); the presence of intraventricular conduction delay (degree of cardiac damage); left ventricular ejection fraction (extent of impairment of left ventricular function); heart rate and serum sodium concentration (measures of activation of the sympathetic nervous system and renin-angiotensin system); and mean arterial pressure and peak oxygen uptake (reflections of the systemic impact of chronic heart failure).

The heart failure survival score was calculated as the absolute value of the sum of the products of the parameters and their computed coefficients:

Score $=\mid[1$ (if coronary artery disease) and 0 (if not) $] \times$ $0.6931+[1$ (if intraventricular conduction delay) and 0 (if not) $] \times 0.6083+($ left ventricular ejection fraction $(\%)) \times$ $-0.0464+$ (heart rate) $\times 0.0216+$ (sodium concentration) $\times-0.0470+($ mean arterial pressure $) \times-0.0255+($ peak oxygen uptake) $\times-0.0546$.

By means of multivariate modelling and definition of arbitrary cut-off points, three groups of disease severity were defined in the derivation cohort for the heart failure survival score (1986-91) and thereafter confirmed in the validation cohort (1993-5). The low risk, medium risk, and high risk patients had a one year event-free survival of $93 \%, 72 \%$, and $43 \%$ respectively in the derivation cohort and $88 \%, 60 \%$, and $35 \%$ in the validation cohort.

For each patient in our study we calculated a heart failure survival score using the same methodology. We used the mean values of the derivation cohort for missing covariates in our COCPIT cohort (see table). We chose this approach in order to use the same cut-off values for the definition of the three risk groups.

\section{Statistical methods}

Patients were followed up to 1 January 2000, giving a minimum follow up of two years after registration. Waiting list outcome consisted of one of the following: transplantation, death on the waiting list, removal from the list because of worsening condition, and removal because of clinical improvement. We used the competing risk method to calculate the probability of each waiting list outcome ${ }^{13} 14$ and the Kaplan-Meier method to analyse the survival rates. To analyse global mortality we ignored whether patients had received a transplant.

Throughout the analysis we tested the null hypothesis of an absence of a difference among the three risk groups versus the alternative hypothesis of a group effect. For outcomes after listing we used the likelihood ratio test. To assess global mortality and differences between groups in outcome after transplantation we used the log rank test.

We applied a time dependent, non-proportional hazard model to cope with the problem of comparing mortality for transplanted patients against that for patients on the waiting list. ${ }^{8}$ This type of modelling is required because the interpretation of this comparison is hampered by a so called "time to event" bias. Thus, if high risk patients were to receive a transplant within days of listing, they would not have time to die on the waiting list, and so a low waiting list mortality would be obtained for this group. If, however, such high risk patients were to receive a transplant after a longer waiting time, they would have had more opportunity to die on the waiting list and would be, by selection, a less sick group at the time of transplantation than the group initially classified as high risk. Consequently, they would have a better outcome after transplantation. Hence, an analysis of patient mortality by treatment modality in which the modality is not fixed in time for all patients in the study needs to take into account the time of switching from the waiting list group to the transplantation group.

Furthermore, this approach allows for transient modelling of the mortality risk after transplantation, which is initially high and then decreases. We reported the transplantation effect as relative risk, which is the ratio of death after transplantation versus the risk of death while on the waiting list for the same period of time. If the relative risk falls below one the risk of dying after transplantation is lower than the risk of dying on the waiting list, which implies that patients benefit from transplantation. If the relative risk remains above one, survival after transplantation is not superior to waiting list survival.

\section{Results}

Patients' baseline characteristics

The table shows the patients' characteristics. The range and distribution of the heart failure survival score was similar in the score's original derivation cohort and in the COCPIT cohort. In our study, 12\%, 41\%, and 47\% of patients were high risk, medium risk, and low risk respectively, compared with $21 \%, 35 \%$, and $44 \%$, in the original derivation cohort.

Patients' characteristics in the derivation cohort for heart failure survival score (HFSS) and in the present cohort (COCPIT study). Values are means (SD) unless stated otherwise

\begin{tabular}{|c|c|c|}
\hline Characteristic & $\begin{array}{l}\text { HFSS derivation cohort } \\
(\mathrm{n}=268)\end{array}$ & $\begin{array}{c}\text { COCPIT cohort } \\
(\mathrm{n}=889)\end{array}$ \\
\hline Age (years) & $50(11)$ & $52(11)$ \\
\hline$\%$ male patients & 80 & 85 \\
\hline New York Heart Association class & $2.8(0.9)$ & $3.3(0.5)$ \\
\hline \multicolumn{3}{|l|}{ HFSS parameters: } \\
\hline Left ventricular ejection fraction (\%) & $20(8)$ & $22(8)$ \\
\hline Peak oxygen uptake $(\mathrm{ml} / \mathrm{min} / \mathrm{kg})$ & $14.6(5.4)$ & $15.8(19.2)^{*}$ \\
\hline Heart rate (beats/min) & $87(15)$ & $85(17)$ \\
\hline Mean arterial pressure $(\mathrm{mm} \mathrm{Hg})$ & $86(13)$ & $82(12)$ \\
\hline Serum sodium concentration $(\mathrm{mmol} / \mathrm{l})$ & $137(4)$ & $138(5)$ \\
\hline$\%$ of patients with intraventricular conduction delay & 27 & NK† \\
\hline$\%$ of patients with ischaemic cardiomyopathy & 45 & 41 \\
\hline Range of HFSS variability & $5.8-10.5$ & $5.6-10.5$ \\
\hline \multicolumn{3}{|l|}{ Severity of heart failure (№ (\%) of patients): } \\
\hline High risk (HFSS $\leqslant 7.19)$ & $56(21)$ & $107(12)$ \\
\hline Medium risk (HFSS=7.20-8.09) & $94(35)$ & $360(41)$ \\
\hline Low risk $(\mathrm{HFSS} \geqslant 8.10)$ & $118(44)$ & $422(47)$ \\
\hline
\end{tabular}

HFSS=heart failure survival score.

COCPIT=comparative outcome and clinical profile in transplantation study.

NK=not known (since COCPIT study started before publication of the heart failure survival score, intraventricular conduction delay was not collected in our cohort).

${ }^{*}$ Incomplete data (139/889). 


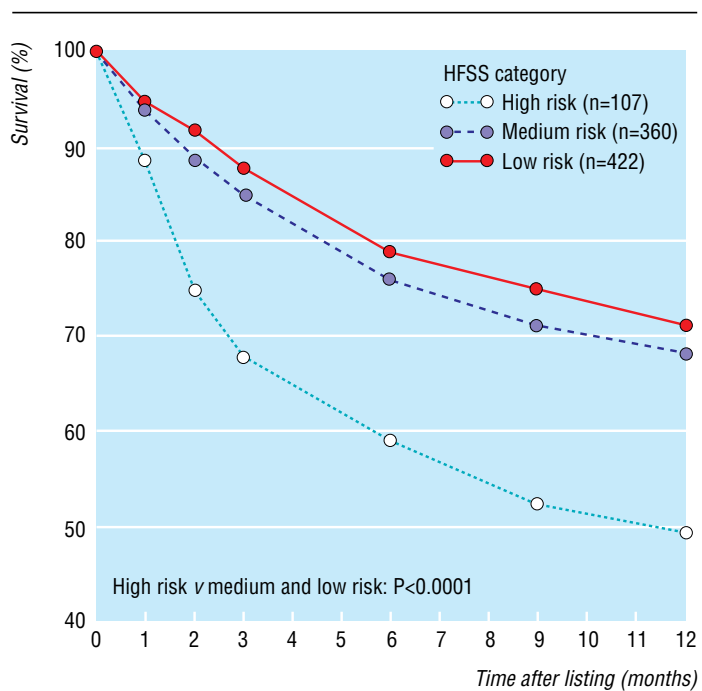

Fig 1 Survival of patients after listing for cardiac transplantation, stratified by heart failure survival score (HFSS)

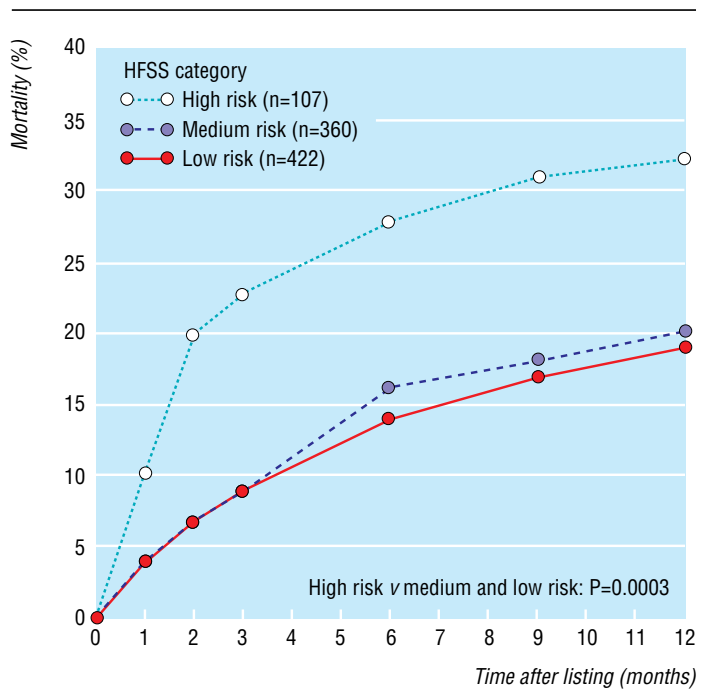

Fig 2 Mortality of patients on waiting list for cardiac transplantation, stratified by heart failure survival score (HFSS)

\section{Outcome after listing}

One year after listing, 33\% of our total cohort had died either before or after transplantation. Those patients who were high risk according to heart failure survival score had a significantly higher chance of dying than patients at medium and low risk ( $51 \%$ v $32 \%$ and $29 \%$ respectively; $\mathrm{P}<0.0001$ ) (fig 1 ).

\section{Waiting list outcome}

One year after registration, 424 patients $(48 \%)$ had undergone cardiac transplantation (358 in the general urgency category, 62 in the special urgency category, and four in the high urgency category); 196 patients $(22 \%)$ had been removed from the list because of death $(\mathrm{n}=182)$ or deterioration $(\mathrm{n}=14) ; 79$ patients $(9 \%)$ had been removed from the list because of improvement in their condition; and 190 (21\%) were still on the waiting list. Figure 2 shows the mortality of patients on the waiting list without transplantation, stratified by heart failure survival score. Twenty per cent of the high risk patients died within two months of listing, and this proportion gradually increased to $32 \%$ at one year after listing. In the medium and low risk groups the probability of dying while waiting was significantly lower $(\mathrm{P}=0.0003)$ and without the initial high mortality seen in the high risk group. The likelihood of transplantation was also significantly higher in the high risk group than in the medium and low risk groups $(\mathrm{P}=0.01)$ : by two months and 12 months of listing respectively, $27 \%$ and $48 \%$ of high risk patients, $17 \%$ and $44 \%$ of medium risk patients, and $11 \%$ and $41 \%$ of the low risk patients had received transplants (fig 3).

\section{Outcome after transplantation}

Survival at one year after transplantation for all patients was $71 \%$ (95\% confidence interval $68 \%$ to $74 \%$ ). Survival at one year for the high risk group was $64 \%$, not significantly different from the $76 \%$ and $75 \%$ for the medium and low risk groups respectively $(\mathrm{P}=0.2)$ (fig 4).

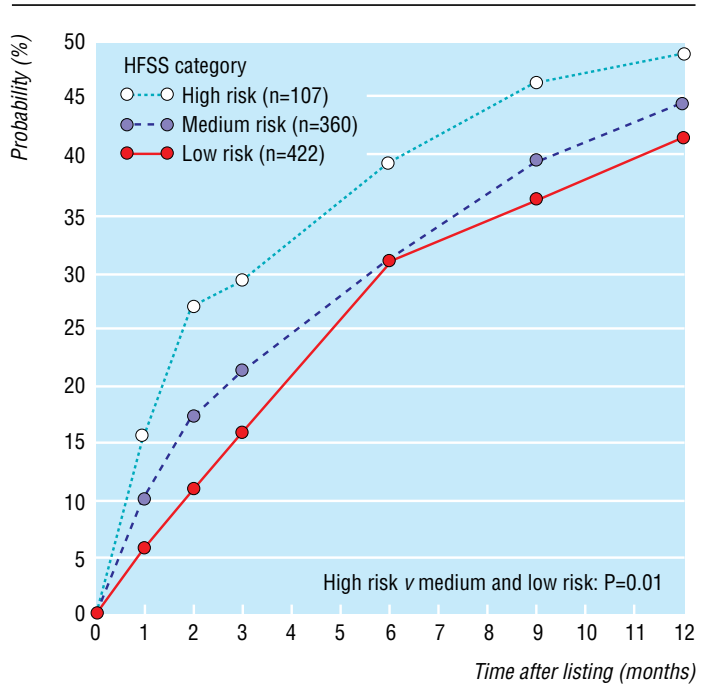

Fig 3 Probability of receiving a transplant for patients after listing for cardiac transplantation, stratified by heart failure survival score (HFSS)

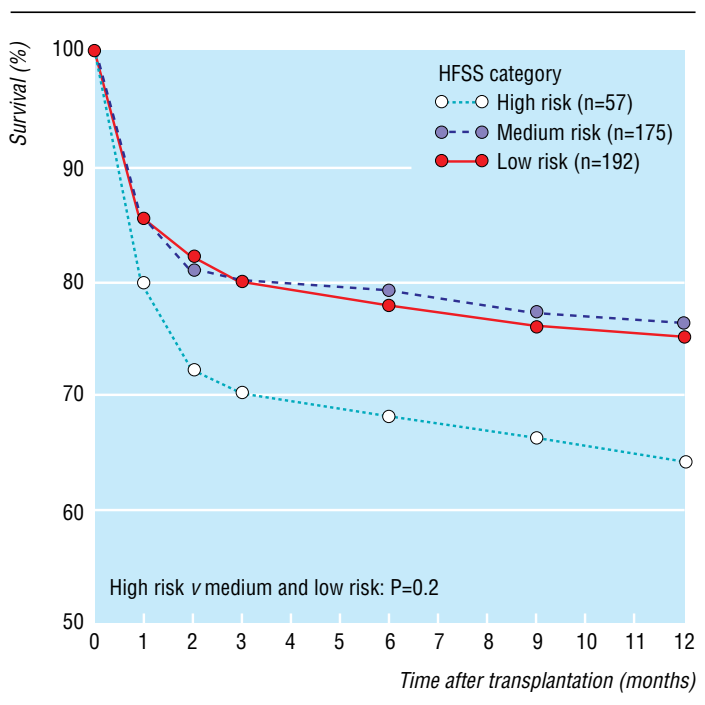

Fig 4 Survival after receiving a cardiac transplant for patients stratified by heart failure survival score (HFSS) 


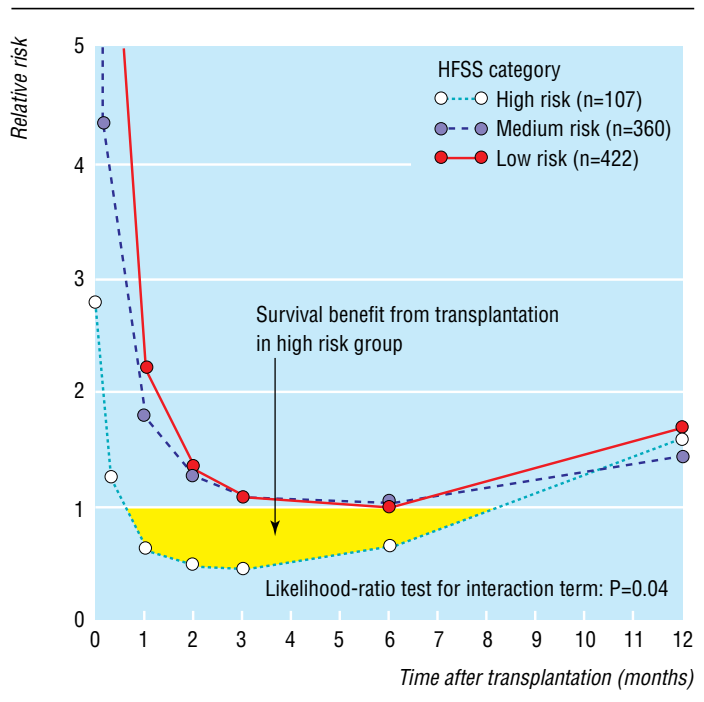

Fig 5 Relative risk of death for patients after receiving a cardiac transplant, stratified by heart failure survival score (HFSS)

\section{Transplantation effect}

For the total cohort there was no survival benefit from transplantation (data not shown). For high risk patients, however, a mortality risk reduction was observed within two weeks of transplantation (relative risk $<1.0$ ). This benefit disappeared after eight months (fig 5). On the other hand, the relative risk did not fall below one for the medium and low risk patients at any time after transplantation.

\section{Discussion}

In this complete national cohort of adult patients consecutively listed for cardiac transplantation in Germany in 1997 no survival benefit accrued from transplantation for the group as a whole. Yet, transplantation had a beneficial effect in the high risk group as early as two weeks after transplantation. Use of the heart failure survival score allowed us to detect differences between risk groups not only with regard to the transplant effect but also with regard to mortality while on the waiting list and global mortality.

\section{Rationale for study}

Since the introduction of cyclosporin in 1980 cardiac transplantation has been considered superior to conventional treatment for heart failure, though the survival benefit of transplantation has never been examined in depth. The Registry of the International Society for Heart and Lung Transplantation reported a one year survival of $78 \%$ in 1999 that had remained constant over the previous five years, ${ }^{6}$ but the Henry Mondor Hospital in Paris, a large and experienced cardiac transplant centre, reported a much lower survival rate at one year after transplantation of $62 \% .^{5}$ An examination of the survival benefit from transplantation is therefore warranted. A randomised trial has been viewed as ethically unacceptable because one of the principles of such trials is that there is genuine uncertainty about the comparative therapeutic merits of each arm in a clinical trial. When the medical community has judged one proposed treatment to be better than another, randomised trials cannot be truly free of bias and may be considered unethical. ${ }^{10}{ }^{15}{ }^{16}$ Furthermore, the application of results from randomised trials is restricted to patients with characteristics similar to those of the cohort examined in the trials. ${ }^{17}$ Therefore, we considered an outcome analysis based on a prospective observational study to be a reasonable approach to test our hypothesis. On the basis of our results, the need for a randomised trial in cardiac transplantation should be debated.

To assess the effect of cardiac transplantation on outcome, a validated scoring system for assessing the clinical severity and associated risk of advanced heart failure is mandatory since any judgment on outcome should take into account the severity of disease. We therefore used the previously validated heart failure survival score to assess risk. Our results confirm the predictive power of the score for risk stratification in this German waiting list cohort. Most importantly, the survival advantage conferred by transplantation on the different risk groups as defined by the score could be assessed with a time dependent, proportional hazards model.

\section{Implications of study}

The high risk patients in our cohort had a global mortality of $51 \%$ at one year after listing. This rate reflects the cumulative mortality of patients on the waiting list and after transplantation. To our knowledge, no other studies have used the same combined end point because patients are usually censored at the time of transplantation. The high global death rate warrants a closer clinical surveillance at the time of listing of high risk patients.

The transient nature of the survival benefit in the high risk group is due to the changing composition of the cohorts who have received a transplant and who are still on the waiting list over time. Once patients have been on the waiting list for several months they become, by selection, a more stable population. All the frail patients have already left the cohort, as a result of either death or transplantation. This observation that survival benefit vanishes as the waiting time lengthens was described in $1991 .^{18}$ Since disease severity is not constant over time, the frequency of re-evaluation of all listed patients should match the current risk of death. It should be higher in high risk states and lower in low risk states.

Our results also imply that the relative importance of medical urgency and waiting time in the listing of patients and allocation to transplantation should be discussed. A policy may opt to allow high risk patients only on the waiting list; this tends to balance organ availability and organ need. Alternatively, all potential candidates can be accepted on the waiting list and subsequently prioritised according to medical urgency, thereby decreasing the impact of waiting time in the allocation algorithm for cardiac transplantation. This change has been suggested by the German Transplantation Society ${ }^{19}$ and the US Department of Health and has been reinforced by the Institute of Medicine of the US National Institutes of Health. ${ }^{20}$

\section{Limitations of study}

Our study cohort had a $71 \%$ survival at one year after transplantation, compared with the $78 \%$ reported by the Registry of the International Society for Heart and Lung Transplantation and the $62 \%$ reported by the 
What is already known on this topic

Heart transplantation is considered the treatment of choice for end stage heart failure because it is assumed to improve survival and quality of life of recipients

This assumption has not been tested in randomised trials or observational cohort studies

\section{What this study adds}

In this complete national German cohort of patients listed for cardiac transplantation during 1997 a survival benefit from transplantation was found only in the subgroup at high risk of dying without transplantation, whereas the medium risk and low risk groups did not derive a survival benefit

These findings challenge the current role of cardiac transplantation in heart failure management and may support the initiative for a randomised trial

Henry Mondor Hospital. Possible explanations for these differences include variations in selection of recipients and donors for heart transplantation, interdisciplinary cooperation, and management protocols as well as considerable variability between centres and regions. The German Transplantation Society has initiated an audit to investigate the influence of these factors. ${ }^{19}$ Whether our results are specific to the German situation or may be generalised to other countries needs to be investigated.

This study did not address the question of whether quality of life was enhanced or cost of treatment reduced by cardiac transplantation. These issues are clearly important and need to be addressed in future studies. Specifically, high risk, medium risk, and low risk patients may benefit to different degrees from cardiac transplantation with regard to quality of life and resource consumption.

\section{Conclusions}

In this complete national cohort of German candidates for cardiac transplantation we found a survival benefit from transplantation only for patients with a predicted high risk of dying on the waiting list. This observation suggests that heart transplantation listing in Germany should currently be limited to the sickest patients. Patients with a predicted low or medium risk had no mortality risk reduction from transplantation; they should instead be managed with organ saving treatments.

We thank Professor G Breithardt for critical comments on the manuscript and Mrs Lilian Streeder for her work in data entry and management. We thank all the participating centres for their enthusiasm and continued support in this national consensus project.

Contributors: MCD jointly initiated the formulation of the primary study hypothesis; coordinated the research; discussed core ideas; jointly designed the protocol, particularly documentation of clinical data; and participated in data collection and analysis and writing the paper. HHS jointly initiated the formulation of the primary study hypothesis; coordinated the research; discussed core ideas; jointly designed the protocol, particularly documentation of clinical data; was instrumental in promoting continued consensus among all German transplant centres; and participated in data analysis and editing the paper. JMJDM jointly initiated the research, discussed essential ideas, was responsible for data collection at the Eurotransplant office and continuously advised from the Eurotransplant perspective, and participated substantially in data analysis and writing the paper. JMAS discussed core ideas of the study hypothesis and design, designed and performed the statistical analysis, and contributed substantially to writing the paper. JH contributed to the statistical analysis of data. All centres mentioned in the appendix contributed to study design, data collection, and critical discussion of the data.

Funding: Eurotransplant International Foundation.

Competing interests: None declared.

\section{Appendix: Participating centres in comparative outcome and clinical profiles in transplantation (COCPIT) study (including chairmen of the} cardiothoracic surgery departments at start of study) Aachen University (Professor B Messmer), Heart Center Bad Krozingen (Dr H Tollenaere), Kerckhoff Center Bad Nauheim (Professor W P Kloevekorn), Heart Center Nordrhein-Westfalia Bad Oeynhausen (Professor R Koerfer), German Heart Center Berlin (Professor R Hetzer), Charite University Berlin (Professor W Konertz), Bochum University (Professor A Laczkovics), Heart Center Dresden (Professor S Schueler), Düsseldorf University (Professor E Gams), Essen University (Professor J C Reidemeister), Frankfurt University (Professor A Moritz), Freiburg University (Professor F Beyersdorf), Heart Center Fulda (Professor T Stegmann), Gießen University (Professor F W Hehrlein), Göttingen University (Professor H Dalichau), Halle University (Professor H R Zerkowski), Hamburg University (Professor R Kalmar), Hannover Medical School (Professor A Haverich), Heidelberg University (Professor S Hagl), Homburg University (Professor H J Schaefers), Heart Center Kaiserslautern (Professor W Seyboldt-Epting), Heart Center Karlsruhe (Dr A Posival), Kiel University (Professor D Regensburger), Köln University (Professor E R De Vivie), Leipzig University (Professor F W Mohr), Mainz University (Professor H Oelert), German Heart Center München (Professor $\mathrm{H}$ Meisner), München University (Professor B Reichart), Münster University (Professor H H Scheld), Regensburg University (Professor D Birnbaum), Tübingen University (Professor G Ziemer), Würzburg University (Professor R E Silber).

1 Hunt SA. 24th Bethesda conference: cardiac transplantation. J Am Coll Cardiol 1993;22:1-64

2 Swedberg K for Consensus Trial Study Group. Effects of enalapril on mortality in severe congestive heart failure. $N$ Engl J Med 1987;316: 1429-35

3 Packer M, Bristow MR, Cohn JN, Colucci WS, Fowler MB, Gilbert EM, et al for the US Carvedilol Heart Failure Study Group. The effect of carvedilol on morbidity and mortality in patients with chronic heart failure. N Engl J Med 1996;334:1349-55.

4 Goldstein DJ, Oz MC, Rose EA. Implantable left ventricular assist devices. N Engl J Med 1998;339:1522-33.

5 Kirsch M, Baufreton C, Naftel DC, Benvenuti C, Loisance DY. Pretransplantation risk factors for death after transplantation: the Henry Mondor experience. J Heart Lung Transplant 1999;17:268-77.

6 Hosenpud JD, Bennett LE, Keck BM, Fiol B, Boucek MM, Novick RJ. The Registry of the International Society for Heart and Lung Transplantation: sixteenth official report-1999. J Heart Lung Transplant 1999;18: 611-26.

7 Edwards EB, Bennett LE, Daily OP, Detre K. Risk of mortality for UNOS status 3 liver recipients: a comparison of the risk posttransplant to the risk on the waiting list. Transplant Proc 1997;29:459-60.

8 Hosenpud JD, Bennett LE, Keck BM, Edwards EB, Novick RJ. Effect of diagnosis on survival benefit of lung transplantation for end-stage lung disease. Lancet 1998;351:24-7.

9 Wolfe RA, Ashby VB, Milford EL, Ojo AO, Ettenger RE, Agodoa LYC, et al. Comparison of mortality in all patients on dialysis, patients on dialysis awaiting transplantation, and recipients of a first cadaveric transplant. $N$ Engl J Med 1999;341:1725-30.

10 Freedman B. Equipoise and the ethics of clinical research. $N$ Engl J Med $1987 ; 317: 141-5$ 
11 Aaronson KD, Schwartz JS, Chen TMC, Wong KL, Goin JE, Mancini DM. Development and prospective validation of a clinical index to predict survival in ambulatory patients referred for cardiac transplant evaluation. Circulation 1997;95:2660-7.

12 Lucey MR, Brown KA, Everson GT, Fung IJ, Gish R, Keefe EB, et al. Minimal criteria for placement of adults on the liver transplant waiting list. Transplantation 1998;66:956-62.

13 Smits JMA, van Houwelingen HC, de Meester JMJ, Persijn GG, Claas FHJ Analysis of the renal transplant waiting list. Application of a parametric competing risk method. Transplantation 1998;66:1146-53.

14 De Meester J, Smits JMA, Persijn GG, Haverich A. Lung transplant waiting list: differential outcome of type of end-stage lung disease, one year after registration. J Heart Lung Transplant 1999;18:563-71.

15 Byar DP, Simon RM, Friedewald WT, Schlesselman J, DeMets DL, Ellenberg JH, et al. Randomized clinical trials. N Engl J Med 1976;295:74-80.
16 Jadad AR, Rennie D. The randomized controlled trial gets a middle-aged checkup. JAMA 1998;279:319-20

17 McGiffin DC, Naftel DC, Kirklin JK, Bourge RC. Advancing knowledge in cardiac transplantation and the place of outcome analysis. Curr Opin Organ Transplant 1998;3:44-50.

18 Stevenson LW, Hamilton MA, Tillisch IH, Moriguchi JD, Kobashigawa J, Creaser JA, et al. Decreasing survival benefit from cardiac transplantation for outpatients as the waiting list lengthens. I Am Coll Cardio for outpatients

19 Deng MC, DeMeester J, Scheld HH. Development of cardiac transplant policy in Germany. Thorac Cardiovasc Surg 2000;48:183-5.

20 Gibbons RD, Meltzer D, Duan N, and other members of the Institute of Medicine Committee on Organ Procurement and Transplantation. Waiting for organ transplantation. Science 2000;287:237-8.

(Accepted 10 August 2000)

\title{
Commentary: Time for a controlled trial?
}

\author{
Tom Treasure, Andrew Murday
}

In their report on cardiac transplantation in Germany Deng et al conclude that, apart from patients with the highest risk of dying on the waiting list, there is no survival advantage. The overall impression given is that there is little to be gained from heart transplantation. This is at odds with the strongly held beliefs of those involved, both doctors and patients. Many transplant recipients are enjoying full and active lives some years after transplantation. Their enjoyment of life and continuing survival are attributed to their new hearts. This difference in perception prompts the question: would a controlled trial resolve the issue?

The German study concentrates on survival at one year, which for transplanted patients was 71\% (95\% confidence interval $68 \%$ to $74 \%$ ). Better survival is reported by the Registry of the International Society for Heart and Lung Transplantation, at $78 \%,{ }^{1}$ and by UK transplant units, at $79 \%$ (77\% to $82 \%)$ (audited nationally under the auspices of the Clinical Effectiveness Unit of the Royal College of Surgeons of England). ${ }^{2}$ Thereafter, the death rate for heart recipients has been found to be constant at about $4 \%$ a year for the next 14 years. ${ }^{1}$ Five year survival in Britain is $66 \%(56 \%$ to $73 \%)$.

In the absence of a controlled trial, what is the best comparison group? The non-transplanted patients on the waiting list selected in the German study are not directly comparable. Furthermore, a comparison at one year is heavily biased against the operated group, who face their highest risk at operation and in the following weeks. In recent clinical trials of heart failure the annual death rate ranged from about $10 \%$ to $20 \%$ and was more or less constant over the two to three years of the trials. $^{3-5}$ Against such a reference population a survival advantage would become evident in the second year and would then widen. The worse the natural course of the selected group the greater the difference in survival.

Not only must comparison of survival be made over a more realistic time span, but there must be recognition of the often dramatic restoration of wellbeing seen in clinical practice and demonstrated objectively. ${ }^{6}$

All the usual obstacles to performing a trial can be raised. Numbers are part of the problem. The German study reports 424 adult cardiac transplant operations among 32 units-that is, 13 cases per unit, barely one a month. In the United States the average unit volume is similar. Currently eight units carry out all the 300 adult heart transplantations performed in Britain, ${ }^{2}$ and we believe that there are good arguments for a reduction rather than an increase in the number of units. Centralisation of resources makes trials easier.

A common ethical obstacle to trials is that treatment believed to be effective is denied to the control group, but many patients deemed clinically suitable are denied a heart transplant because of the limited number of donor organs. This makes transplantation unusual in that there is an explicit external constraint that limits volume, however much funding were to be made available. It follows that if we are to get the maximum benefit out of the limited number of donor hearts we should use them where the most quality years can be gained. A strategy aimed at maximising the benefit gained by each donor organ may be more logical than selecting the most desperate recipients, the policy which would flow from the interpretation of the German data. If there is real doubt about where maximum benefits can be gained the best way to resolve this is by clinical trial. There are insufficient organs to go round, ${ }^{7}$ and once beliefs about case selection are challenged by reports such as this one from Germany it may be as ethical to allocate the limited supply of hearts within carefully designed clinical trials as by the clinical decisions of individuals.

1 Hosenpud JD, Bennett LE, Keck BM, Fiol B, Boucek MM, Novick RJ. The Registry of the International Society for Heart and Lung Transplantation: sixteenth official report-1999.J Heart Lung Transplant 1999;18:611 26.

2 Anyanwu AC, Rogers CA, Murday AJ. Review of the current state of thoracic transplantation: a national prospective cohort study. Transplant Proc $1999 ; 31: 165$

3 The cardiac insufficiency bisoprolol study II (CIBIS-II): a randomised trial. Lancet 1999;353:9-13.

4 Effect of metoprolol CR/XL in chronic heart failure: metoprolol CR/XI randomised intervention trial in congestive heart failure (MERIT-HF) Lancet 1999:353:2001-7.

5 Pitt B, Zannad F, Remme WJ, Cody R, Castaigne A, Perez A, et al. The effect of spironolactone on morbidity and mortality in patients with severe heart failure. Randomized Aldactone Evaluation Study Investigators. N Engl J Med 1999;341:709-17.

6 O'Brien BJ, Buxton MJ, Ferguson BA. Measuring the effectiveness of heart transplant programmes: quality of life and their relationship to survival analysis. J Chromic Dis 1987:40(suppl 1):137-58S.

7 Organ donation in the 21 st century. London: BMA, 2000.
St George's

Hospital Medical School, St George's Hospital, London SW17 0QT

Tom Treasure professor of cardiothoracic surger Andrew Murday consultant cardiac surgeom

Correspondence to: T Treasure Tom.Treasure@ ukgateway.net 